Research Paper

\title{
A Noninvasive Assessment of Tumor Proliferation in Lung cancer Patients using Intravoxel Incoherent Motion Magnetic Resonance Imaging
}

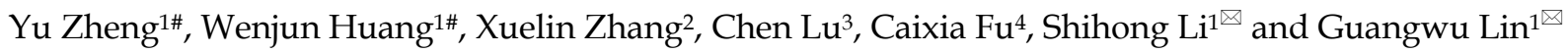 \\ 1. Department of Radiology, Huadong Hospital Affiliated to Fudan University, Shanghai, 200040, China. \\ 2. Department of Thoracic Surgery, Huadong Hospital Affiliated to Fudan University, Shanghai, 200040, China. \\ 3. Department of Pathology, Huadong Hospital Affiliated to Fudan University, Shanghai, 200040, China. \\ 4. Siemens Shenzhen Magnetic Resonance Ltd., Shenzhen, Guangdong Province, 518057, China. \\ \#Equal contributions of co-first authors. \\ $\triangle$ Corresponding authors: Shihong Li (E-mail: lishihong12@fudan.edu.cn), Guangwu Lin (E-mail: lingw01000@163.com).
}

(1) The author(s). This is an open access article distributed under the terms of the Creative Commons Attribution License (https://creativecommons.org/licenses/by/4.0/). See http://ivyspring.com/terms for full terms and conditions.

Received: 2020.05.23; Accepted: 2020.10.26; Published: 2021.01.01

\begin{abstract}
$\mathrm{Ki}-67$ is a nuclear antigen widely used in routine pathologic analyses as a tumor cell proliferation marker for lung cancer. However, Ki-67 expression analyses using immunohistochemistry (IHC) are invasive and frequently influenced by tissue sampling quality. In this study, we assessed the feasibility of noninvasive magnetic resonance imaging (MRI) in predicting the Ki-67 labeling indices (LIs). A total of 51 lung cancer patients, including 42 non-small cell lung cancer (NSCLC) cases and nine small cell lung cancer (SCLC) cases, were enrolled in this study. Quantitative MRI parameters from conventional diffusion-weighted imaging (DWI), intravoxel incoherent motion (IVIM), and diffusion kurtosis imaging (DKI) were obtained, and their correlations with tumor tissue Ki-67 expression were analyzed. We found that the true diffusion coefficient ( $D$ value) from IVIM was negatively correlated with Ki-67 expression (Spearman $r=-0.76, P<0.001$ ). The $D$ values in the high $\mathrm{Ki}-67$ group were significantly lower than those in the low Ki-67 group $\left(0.90 \pm 0.21 \times 10^{-3} \mathrm{~mm}^{2} / \mathrm{s}\right.$ vs. $1.22 \pm 0.30$ $\times 10^{-3} \mathrm{~mm}^{2} / \mathrm{s}$ ). Among three MRI techniques used, D values from IVIM showed the best performance for distinguishing the high $\mathrm{Ki}-67$ group from low $\mathrm{Ki}-67$ group in receiver operating characteristic (ROC) analysis with an area under the ROC curve (AUROC) of 0.85 (95\% Cl: 0.73-0.97, $P<0.05)$. Moreover, $D$ values performed well for differentiating SCLC from NSCLC with an AUROC of 0.82 ( $95 \% \mathrm{Cl}: 0.68-0.90)$, Youden index of 0.72 , and $\mathrm{Fl}$ score of 0.81 . In conclusion, $\mathrm{D}$ values were negatively correlated with $\mathrm{Ki}-67$ expression in lung cancer tissues and can be used to distinguish high from low proliferation statuses, as well as SCLC from NSCLC.
\end{abstract}

Key words: IVIM; Ki-67; MRI; NSCLC; SCLC; tumor differentiation

\section{Introduction}

Lung cancer was the most frequently diagnosed cancer and the leading cause of cancer death among males in 2012 [1]. Lung cancer survival rates are poor, with five-year survival rates of less than $20 \%$ [2]. Ki-67 is a nuclear antigen present in most proliferating cells. $\mathrm{Ki}-67$ is expressed during the active phases of the cell cycle, including the G1, G2, and S phases, and is a common marker used to detect tumor cell proliferation related to tumor invasiveness and prognoses. The Ki-67 labeling index (LI) has been widely used to predict the prognoses of breast cancer [3], glioma [4], and lung cancer [5]. A meta-analysis demonstrated that high Ki-67 expression is associated with poor prognoses and disease progression in lung cancer patients. Ki-67 has been used as an independent biologic marker to predict lung cancer patient prognoses [6]. However, the quantification of Ki-67 expression using immunohistochemistry (IHC) is invasive and frequently influenced by tissue sampling quality [7].

The application of magnetic resonance imaging (MRI) to lung cancer analyses is a relatively recent development yet is a rapidly growing field. Apparent diffusion coefficient (ADC) values derived from 
diffusion-weighted imaging (DWI) can reflect tumor cellularity. In recent years, ADC values were reported to be associated with $\mathrm{Ki}-67$ expression in various tumors, such as breast cancer [8, 9], endometrial stromal sarcoma of the uterus [10], endometrial carcinoma [11], and lung cancer [12]. However, ADC values are calculated using a mono-exponential model that is affected not only with the molecular movements of water but also by capillary microcirculation [13].

Intravoxel incoherent motion (IVIM) allows the separation of perfusion and molecular-based diffusion coefficients [14]. By fitting multiple-b DWI data with a biexponential model, three parameters can be obtained from IVIM, including the true diffusion coefficient (D value), perfusion-related pseudodiffusion coefficient $\left(\mathrm{D}^{*}\right.$ value), and perfusion fraction (f value) [15-18]. In addition, tumor tissue microenvironments are complicated, making the diffusion of water molecules behave in a nonGaussian fashion, especially when b values are $>1000$ $\mathrm{s} / \mathrm{mm}^{2}$ [19]. A non-Gaussian diffusion model, diffusion kurtosis imaging (DKI), proposed by Jensen et al. [20], can measure tissue structures, including cellular compartments and membranes [18, 21-23].

To our knowledge, no studies have evaluated the associations between the quantitative parameters derived from IVIM and DKI and Ki-67 expression in lung cancer tissues. The difference in these IVIM and DKI parameters between small cell lung cancer (SCLC) and non-small cell lung cancer (NSCLC) are also largely unknown. Therefore, this study aimed to evaluate whether IVIM and DKI can predict Ki-67 expression levels in lung cancer tissues preoperatively and if IVIM and DKI quantitative values differ between SCLC and NSCLC.

\section{Materials and methods}

\section{Patients}

This prospective study was approved by the Clinical Research Ethics Committee of Huadong Hospital, Fudan University. All procedures performed in studies involving human participants were in accordance with the ethical standards of the institutional and/or national research committee and with the 1964 Helsinki Declaration and its later amendments or comparable ethical standards. Informed consent was obtained from all individual participants involved in the study.

From September 2016 to August 2018, patients who met the following criteria were included: (1) pulmonary nodule or mass diameters larger than 15 $\mathrm{mm}$ as detected by computed tomography (CT); (2) $>50 \%$ of the tumors were solid; (3) Ki-67 tumor expression was available; (4) no previous treatments were given before MR examinations. The exclusion criteria were: (1) MRI contraindications present $(\mathrm{n}=$ 13); (2) unsatisfactory image qualities with server motion or distortion artifacts $(n=9)$; (3) A lack of pathologic results $(n=8)$. A total of 51 lung cancer patients were finally enrolled in this study, with 42 NSCLC and 9 SCLC patients. The characteristics of the enrolled patients are shown in Table 1.

\section{MRI acquisitions}

All MRI examinations were performed with a 3-T MR scanner (MAGNETOM Prisma, Siemens Healthcare, Erlangen, Germany) using a 32-channel body coil and an integrated spine coil. After routine scanning that included coronal and transverse half acquisition single-shot turbo spin-echo (HASTE) T2-weighted imaging (T2WI), transversal turbo spin-echo (TSE) T2WI with fat suppression, and T1-volumetric interpolated breath-hold examination (VIBE), multi-b diffusion-weighted MRI scans $(b=0$, 20, 60, 80, 150, 200, 400, 600, 800, 1200, 1600, 2000 $\mathrm{s} / \mathrm{mm}^{2}$ ) with a single-shot echo-planar imaging pulse sequence in an axial orientation during free breathing was performed. The MRI protocols were listed in Table S1.

\section{Image analyses}

All quantitative derived parameter maps were calculated using the prototype software Body Diffusion Toolbox (Siemens Healthcare, Erlangen, Germany). The DWI data were respectively postprocessed with the mono-exponential and biexponential models. The ADC was calculated using the mono-exponential model from DWI with $b$ values of 0 and $800 \mathrm{~s} / \mathrm{mm}^{2}$, as show below:

$$
S(b)=S_{0} \exp (-b A D C)
$$

where $S(b)$ represents the signal intensities at a specified $\mathrm{b}$ value, and $\mathrm{S}_{0}$ represents the signal intensities measured without radiofrequency saturations. Tumor regions of interest (ROIs) were drawn by outlining the tumor borders on ADC maps and showing the largest cross-section of the tumors. Necrotic areas and adjacent large vessels were avoided. Then the software-generated mean ADC values were recorded.

The IVIM parameters were calculated by fitting the acquired signal with $9 \mathrm{~b}$-values $(0,20,60,80,150$, $200,400,600$, and $800 \mathrm{~s} / \mathrm{mm}^{2}$ ) into the IVIM model equation described by Le Bihan et al. [14]:

$$
S(b)=S_{0}\left[(1-f) \exp (-b D)+f \exp \left(-b D^{*}\right)\right.
$$

where $\mathrm{D}^{*}$ is the pseudo-diffusion coefficient representing the perfusion-related incoherent microcirculation, $\mathrm{f}$ is the pseudodiffusion fraction, 
and $\mathrm{D}$ is the true diffusion coefficient representing pure molecular diffusion.

DKI parameters including Dapp and Kapp, were obtained with six $b$-value signal intensities $(b=0,600$, $800,1200,1600$, and $2000 \mathrm{~s} / \mathrm{mm}^{2}$ ) fitted into the following equation [20]:

$$
S(b)=S_{0} \exp \left(-b D a p p+\frac{b^{2} D a p p^{2} K a p p}{6}\right)
$$

where Dapp represents diffusivity, and Kapp represents diffusion kurtosis.

Two radiologists (with 6 and 10 years of experience in MRI, respectively), who were blinded to the pathologic results, drew ROIs on the ADC maps and recorded the values of each parameter independently. The ROIs were automatically copied from the ADC maps to the corresponding IVIM-DKI parametric maps to obtain the values of D, D*, f, Dapp, and Kapp. Each lesion ROI was drawn twice and averaged to a mean value for analyses.

All DWI parameters were performed using a prototype Body Diffusion Toolbox (Siemens Healthcare, Erlangen, Germany). We selected 9 b-values $(0,20,60,80,150,200,400,600$, and 800 $\mathrm{s} / \mathrm{mm}^{2}$ ) to calculate DWI and IVIM parameters with the mono- and bi-exponential models, respectively. Six b-values $(b=0,600,800,1200,1600$ and 2000 $\mathrm{s} / \mathrm{mm}^{2}$ ) were selected for the DKI parameter calculations. The two blinded radiologists performed the following measurements. Tumor regions of interest (ROIs) were drawn by outlining tumor borders on ADC maps, showing the largest tumor cross-sections, and avoiding necrotic areas and adjacent large vessels by referring to T2WI and DWI images. The same ROIs were automatically copied to the $\mathrm{D}, \mathrm{D}^{*}, \mathrm{f}$, Dapp, and Kapp maps at the same level. All DWI parameters were measured synchronously.

\section{Immunohistochemistry}

The immunohistochemical (IHC) analyses of Ki-67 expression were performed using the mouse monoclonal anti-human Ki-67 antibody (MIB-1, ZSGBBIO, Beijing, China). A pathologist with 14 years of experience in lung cancer pathology, blinded to the clinical and MRI data, assessed Ki-67 tumor expression. The percentage of Ki-67-positive cells was assessed by counting the number of stained nuclei per 100 tumor cells in the most representative areas $(\times 400)$, corresponding to areas with the highest mitotic activity. Then, Ki-67 expression levels were divided into low $(\leq 25 \%)$ and high $(>25 \%)$ Ki67 expression groups, based on previous studies [24-26].

\section{Statistical analyses}

Statistical analyses were conducted using SPSS 22.0 (IBM SPSS Statistics, USA) or GraphPad Prism 8.0
(Prism, USA) software. Quantitative data were expressed as the mean \pm standard deviation (SD) or median and interquartile ranges based on the distribution. The interclass correlation coefficient (ICC) was used to evaluate reader reproducibility for parameter measurements $(0.00-0.20$, poor correlation; 0.21-0.40, fair correlation; 0.41-0.60, moderate correlation; 0.61-0.80, good correlation; and 0.81-1.00, excellent correlation). The correlations between the DWI-derived parameters and Ki-67 expression were analyzed using Spearman's rank correlations. The differences in DWI parameters between low and high Ki-67 expression and between SCLC and NSCLC were analyzed with a Student's t-test or MannWhitney U-test. Receiver operating characteristic (ROC) curve analyses were performed to determine the optimal cut-off values of these parameters for predicting high Ki-67 expression levels and lung cancer subtypes. The area under the ROC curve (AUCROC), sensitivity, specificity, Youden index, and F1 score were calculated. A $P<0.05$ was considered statistically significant.

\section{Results}

\section{Patients' characteristics}

A total of 51 lung cancer patients were enrolled in this study, including 42 NSCLC and 9 SCLC patients. The median age was 64 (Range: 42-83). The mean Ki-67 values of all 51 patients was $39.7 \pm 26.9 \%$ (range: 2\%-90\%). Thirty-one patients had Ki-67 expression values of $>25 \%$ (Table 1 ).

Table 1. Characteristics of patients in this study

\begin{tabular}{|c|c|c|c|}
\hline Characteristics & Value & Percentage & $P$ value \\
\hline \multicolumn{4}{|l|}{ Sex } \\
\hline Male & 27 & $52.9 \%$ & 0.69 \\
\hline Female & 24 & $47.1 \%$ & \\
\hline \multicolumn{4}{|l|}{ Age } \\
\hline Median (range) & $64(42-83)$ & & \\
\hline Ki67 (Mean \pm SD) & $39.7 \pm 26.9$ & & \\
\hline$>25 \%(\mathrm{n})$ & 31 & $60.8 \%$ & 0.05 \\
\hline$\leq 25 \%(\mathrm{n})$ & 20 & $39.2 \%$ & \\
\hline \multicolumn{4}{|l|}{ Pathological feature } \\
\hline NSCLC (Non-small cell lung cancer) & 42 & $82.4 \%$ & \\
\hline Adenocarcinoma & 28 & & \\
\hline Squamous cell carcinoma & 12 & & $<0.001$ \\
\hline Large cell carcinoma & 2 & & \\
\hline SCLC (Small cell lung cancer) & 9 & $17.7 \%$ & \\
\hline
\end{tabular}

\section{DWI images and ADC, IVIM, and DKI maps of lung tumors in patients with NSCLC and SCLC}

The diffusion-weighted images of a typical NSCLC patient are shown in Figure S1. Tumor signals 
were attenuated with ascending $b$ values. Figure 1 shows representative parametric maps of a 58-yearold male diagnosed with SCLC. The parametric maps (ADC, IVIM, and DKI) of an 80-year-old male diagnosed with lung adenocarcinoma are shown in Figure 2. Figures S2 and S3 show a 75-year-old male patient with lung squamous cell carcinoma and a 72-year-old male patient with large cell carcinoma, respectively. The parametric maps are followed by images of hematoxylin-eosin and Ki-67 immunohistochemical stained tissue sections.

\section{The associations between the quantitative IVIM and DKI values and Ki-67 expression}

The interclass correlation coefficients (ICCs) for $D^{*}$ ranged from $0.33-0.84$, and the ICCs of other parameters were $0.701-0.905$. The $\mathrm{ADC}, \mathrm{D}$, and $\mathrm{D}^{*}$ values of the high Ki-67 group were $1.11 \pm 0.26 \times 10^{-3}$ $\mathrm{mm}^{2} / \mathrm{s}, 0.90 \pm 0.21 \times 10^{-3} \mathrm{~mm}^{2} / \mathrm{s}, 16.66 \pm 8.07 \times 10^{-3}$ $\mathrm{mm}^{2} / \mathrm{s}$, respectively, and were significantly lower than low Ki-67 group (ADC, D and $\mathrm{D}^{*}$ values were $1.33 \pm 0.30 \times 10^{-3} \mathrm{~mm}^{2} / \mathrm{s}, 1.22 \pm 0.30 \times 10^{-3} \mathrm{~mm}^{2} / \mathrm{s}$, $23.09 \pm 12.70 \times 10^{-3} \mathrm{~mm}^{2} / \mathrm{s}$, respectively) (Table 2 ). However, the Kapp value of the high Ki-67 group $(0.78 \pm 1.98)$ was significantly higher than that of the low Ki-67 group $(0.64 \pm 0.15)$ (Table 2 and typical cases shown in Figures 1 and 2, and Figures S2 and S3). There were no significant differences in the $f$ and Dapp values between the high and low Ki-67 groups.

Table 2. Comparison of ADC, D, D*, f, Kapp, and Dapp values of lung tumors with low and high Ki-67

\begin{tabular}{llll}
\hline Parameters & Low Ki-67 $(\mathrm{n}=21)$ & High Ki-67 $(\mathrm{n}=30)$ & $P$ \\
\hline ADC $\left(\times 10^{-3} \mathrm{~mm}^{2} / \mathrm{s}\right)$ & $1.33 \pm 0.30$ & $1.11 \pm 0.26$ & 0.012 \\
$\mathrm{D}\left(\times 10^{-3} \mathrm{~mm}^{2} / \mathrm{s}\right)$ & $1.22 \pm 0.30$ & $0.90 \pm 0.21$ & 0.000 \\
$\mathrm{D}^{*}\left(\times 10^{-3} \mathrm{~mm}^{2} / \mathrm{s}\right)$ & $23.1 \pm 12.7$ & $16.7 \pm 8.07$ & 0.031 \\
$\mathrm{f}(\%)$ & $30.3 \pm 15.2$ & $26.9 \pm 16.5$ & 0.48 \\
Kapp & $0.64 \pm 0.15$ & $0.78 \pm 1.98$ & 0.011 \\
Dapp $\left(\times 10^{-3} \mathrm{~mm} / \mathrm{s}\right)$ & $1.91 \pm 0.51$ & $1.74 \pm 0.68$ & 0.34 \\
\hline
\end{tabular}

Data are mean \pm standard deviation (SD). ADC: apparent diffusion coefficient; D: true diffusion coefficient, $\mathrm{D}^{*}$ value: the perfusion-related pseudodiffusion coefficient; f: perfusion fraction; Kapp: diffusion kurtosis; Dapp: diffusivity.
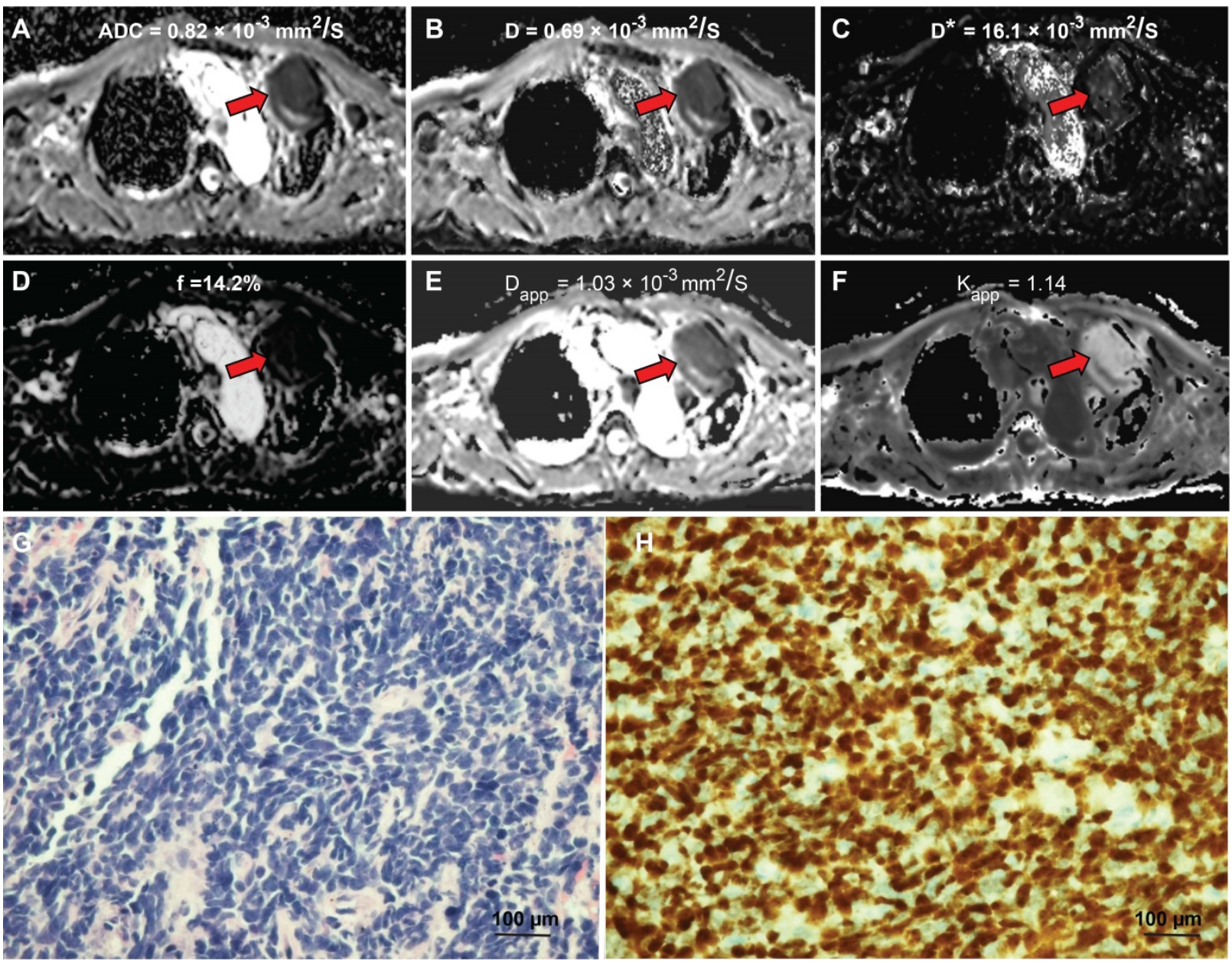

Figure 1. A 58-year-old male diagnosed with small cell lung cancer. (A) An axial ADC map showed a hypointense mass in the left pulmonary lobe (red arrow), with an ADC value of $0.82 \times 10^{-3} \mathrm{~mm}^{2} / \mathrm{s}$. (B) A diffusion map (D) demonstrating a reduced $D$ value $\left(0.69 \times 10^{-3} \mathrm{~mm}^{2} / \mathrm{s}\right)$. (C) A pseudodiffusion coefficient (D*) map demonstrating a $D^{*}$ value of $16.01 \times 10^{-3} \mathrm{~mm}^{2} / \mathrm{s}$. (D) A perfusion fraction ( $\mathrm{f}$ map showing an $\mathrm{f}$ value of $14.17 \%$. (E) A diffusion map (Dapp) showing a Dapp value of $1.03 \times 10^{-3} \mathrm{~mm} / \mathrm{s}$. (F) A kurtosis map showing a Kapp value of 1.14. (G) Small cell lung cancer was confirmed by Hematoxylin and eosin (H\&E) staining (magnification, $\times 400 ;$ scale bar, $100 \mu$ m). (H) Ki-67 immunohistochemical labeling shows that approximately $90 \%$ of cells are positive for nuclear staining (magnification, $\times 400$, scale bar, $100 \mu$ m). ADC: Apparent diffusion coefficient; D: true diffusion coefficient, D* value: the perfusion-related pseudodiffusion coefficient; f: perfusion fraction; Kapp: diffusion kurtosis; Dapp: diffusivity. 

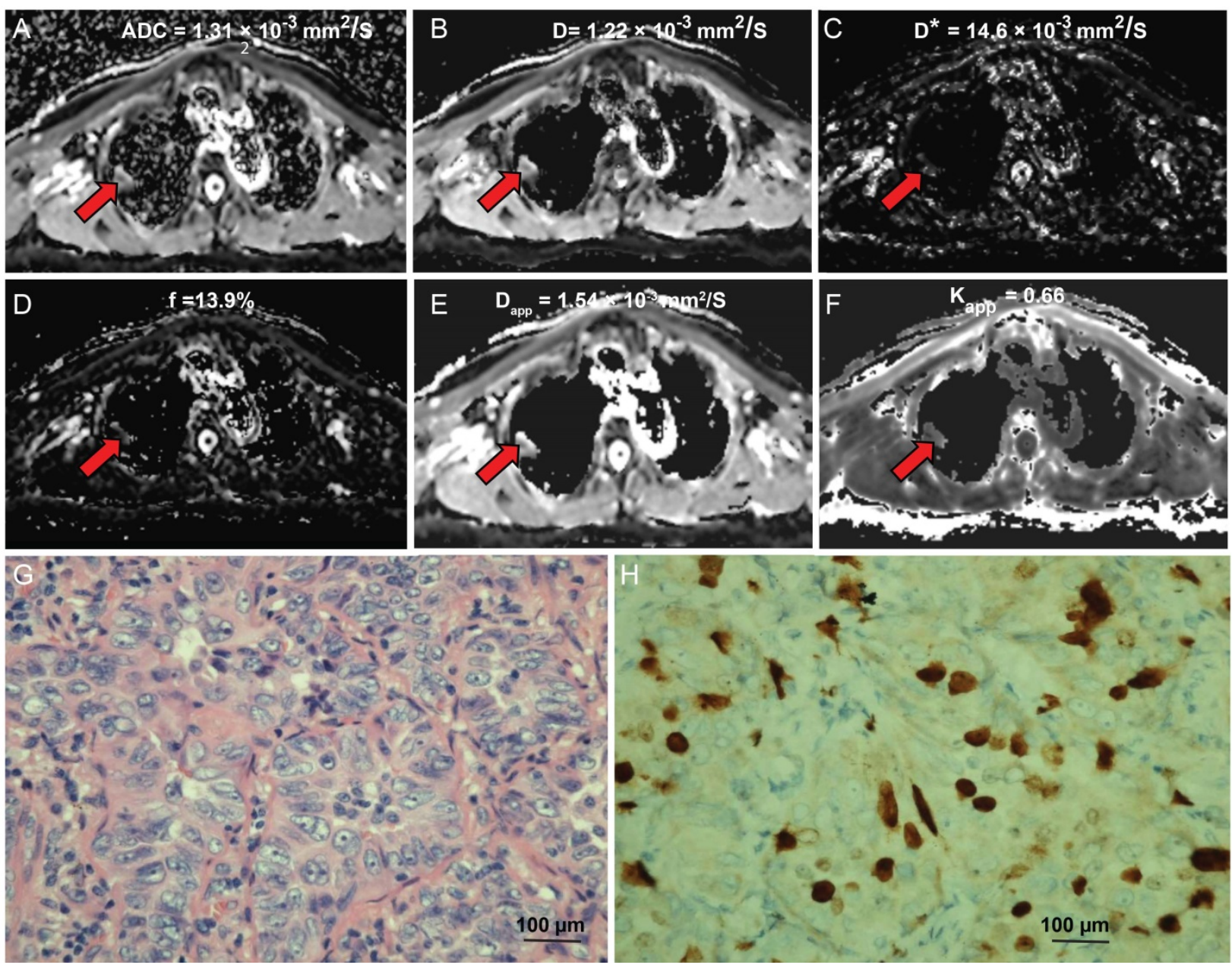

Figure 2. An 80-year-old male diagnosed with lung adenocarcinoma. (A) An axial ADC map shows a hypointense mass in the left pulmonary lobe (red arrow), with an $A D C$ value of $1.13 \times 10^{-3} \mathrm{~mm}^{2} / \mathrm{s}$. (B) A diffusion map demonstrating a $D$ value of $1.22 \times 10^{-3} \mathrm{~mm}^{2} / \mathrm{s}$. (C) A pseudodiffusion coefficient map demonstrating a $D^{*}$ value of $14.63 \times 10^{-3}$ $\mathrm{mm}^{2} / \mathrm{s}$. (D) A perfusion fraction map showing an $\mathrm{f}$ value of $13.94 \%$. (E) A diffusion map showing a Dapp value of $1.54 \times 10^{-3} \mathrm{~mm}^{2} / \mathrm{s}$. (F) A kurtosis map showing a Kapp value of 0.66 . (G) Hematoxylin and eosin (H\&E) staining confirms the mass to be lung adenocarcinoma (magnification, $\times 400$, scale bar, $100 \mu \mathrm{m})$. (H) Ki-67 immunohistochemical labeling shows that approximately $10 \%$ of cells are positive for nuclear staining (magnification, $\times 400$, scale bar, $100 \mu \mathrm{m}$ ).

A

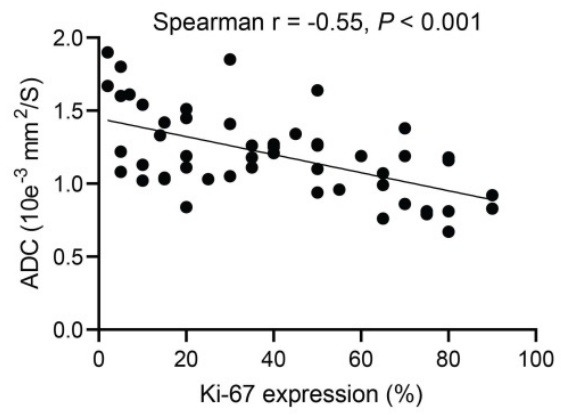

B

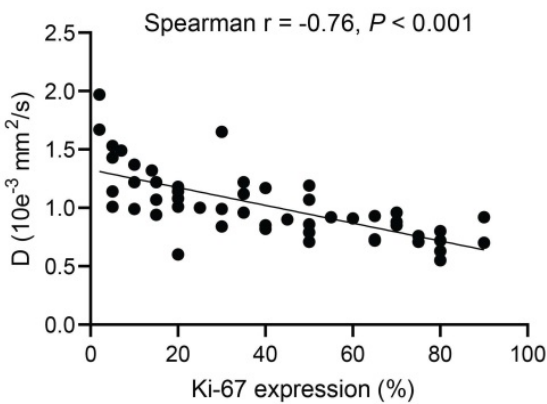

C

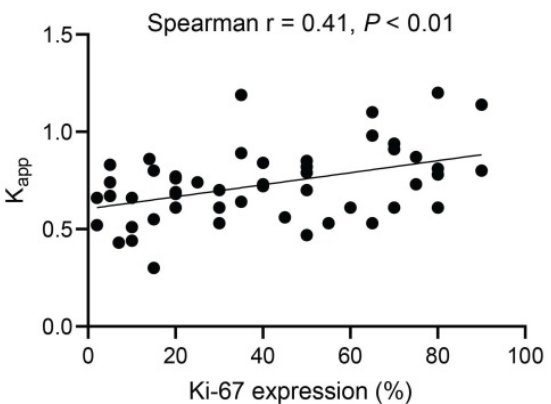

Figure 3. The significant correlations between the quantitative values of different magnetic resonance imaging (MRI) techniques and Ki-67 expression in lung tumor tissue sections. (A) Apparent diffusion coefficient (ADC); (B) True diffusion coefficient (D value); (C) Kapp values (diffusion kurtosis) from diffusion kurtosis imaging (DKI). Spearman's rank correlation was performed.

Spearman's rank correlation analyses revealed that the ADC and D values were negatively correlated with Ki-67 expression ( $\mathrm{r}=-0.55$ and -0.76 , respectively, all $P<0.001$ ) (Figures $3 \mathrm{~A}$ and 3B). In contrast, Kapp values were positively correlated with Ki-67 expression $(\mathrm{r}=0.41, P<0.01)$ (Figure $3 \mathrm{C})$. No significant correlations were found between $D^{*}, f$, Dapp, and Ki-67 expression (Table S2).

The performance of ADC, D, D*, Dpp, and Kpp for distinguishing high $\mathrm{Ki}-67$ group from the low Ki-67 group was then tested using AUROC curve analyses. Among the tested parameters, $\mathrm{D}$ values had 
the highest AUC of 0.85 (95\% CI: 0.73-0.97, $P<0.001$ ) in discriminating the high $\mathrm{Ki}-67$ group from the low Ki-67 expression group (Figure 4A). In addition, D values yielded a sensitivity of $90.2 \%$, a specificity of $77.4 \%$, and a Youden index of 0.67 (Table 3 ).

\section{Quantitative MRI assessments for distinguishing SCLC and NSCLC}

We found that the ADC, D, and Dapp values of SCLC were significantly lower than those of NSCLC (All $P$ values $<0.05$ ) (Table S3). In contrast, the Kapp values of SCLC were significantly higher than those of NSCLC $(0.90 \pm 0.29$ vs. $0.69 \pm 0.14, P=0.048)$. There were no significant differences in $D^{*}$ and $f$ values between the two lung cancer types (all $P>0.05$ ).

$D$ values (Cutoff value $=0.85$ ) performed well in discriminating SCLC and NSCLC with an AUC of 0.82 (95\% CI: 0.68-0.92) (Figure 4B and Table S4). Compared with ADC, Dapp, and Kapp values, D values from IVIM yielded higher Youden index values (Table S4).

\section{Discussion}

Differentiating SCLC from NSCLC (large cell carcinoma or basaloid squamous cell carcinoma) using noninvasive methods is essential since the therapeutic strategies and clinical prognoses are significantly different. Several noninvasive blood metabolomic and dynamic multiphase computed tomography (CT) methods have been recently developed to discriminate SCLC and NSCLC [27-30]. However, blood metabolomic biomarker results that showed the differentiation of SCLC and NSCLC tumor types has not been confirmed with large cohort studies. Dynamic multiphase CT was shown to distinguish SCLC and NSCLC by evaluating tumor perfusion; however, significant radiation exposures restrict the use of this methodology. As a noninvasive, reusable technique, quantitative MRI is frequently used for tumor evaluations. In this study, we demonstrated for the first time that $\mathrm{D}$ values from IVIM had excellent accuracy in distinguishing NSCLC and SCLC.

Table 3. ROC curve analysis for ADC, D, D*, and Kapp values in discriminating lung cancers with high and low Ki-67 expression

\begin{tabular}{|c|c|c|c|c|c|c|}
\hline Parameters & Cut-off value & Sensitivity (\%) & Specificity (\%) & AUROC (95\% CI) & Youden index & $P$ \\
\hline $\mathrm{ADC}\left(\times 10^{-3} \mathrm{~mm}^{2} / \mathrm{s}\right)$ & 1.42 & 45.1 & 93.5 & $0.68(0.53-0.84)$ & 0.39 & 0.029 \\
\hline $\mathrm{D}\left(\times 10^{-3} \mathrm{~mm}^{2} / \mathrm{s}\right)$ & 0.98 & 90.2 & 77.4 & $0.85(0.73-0.97)$ & 0.67 & 0.000 \\
\hline $\mathrm{D}^{*}\left(\times 10^{-3} \mathrm{~mm}^{2} / \mathrm{s}\right)$ & 13.1 & 85.2 & 45.2 & $0.65(0.49-0.81$ & 0.31 & 0.073 \\
\hline Kapp & 0.78 & 51.6 & 85 & $0.69(0.55-0.83)$ & 0.37 & 0.023 \\
\hline
\end{tabular}

ROC: receiver operating characteristic; ADC: apparent diffusion coefficient; $D$ : true diffusion coefficient, $\mathrm{D}^{*}$ value: the perfusion-related pseudodiffusion coefficient; $\mathrm{f}$ : perfusion fraction; Kapp: diffusion kurtosis; Dapp: diffusivity; AUROC: Area under ROC curve; CI: confidence interval.

A

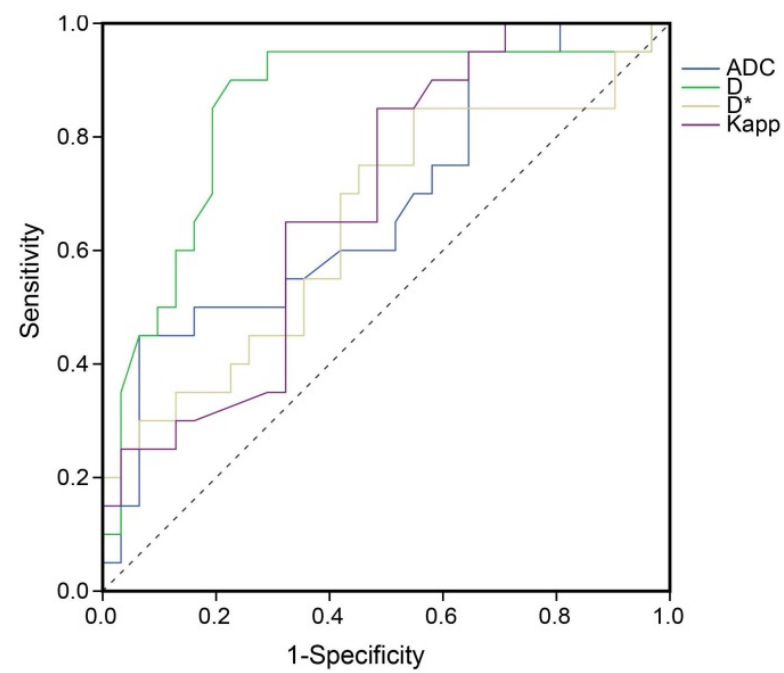

B

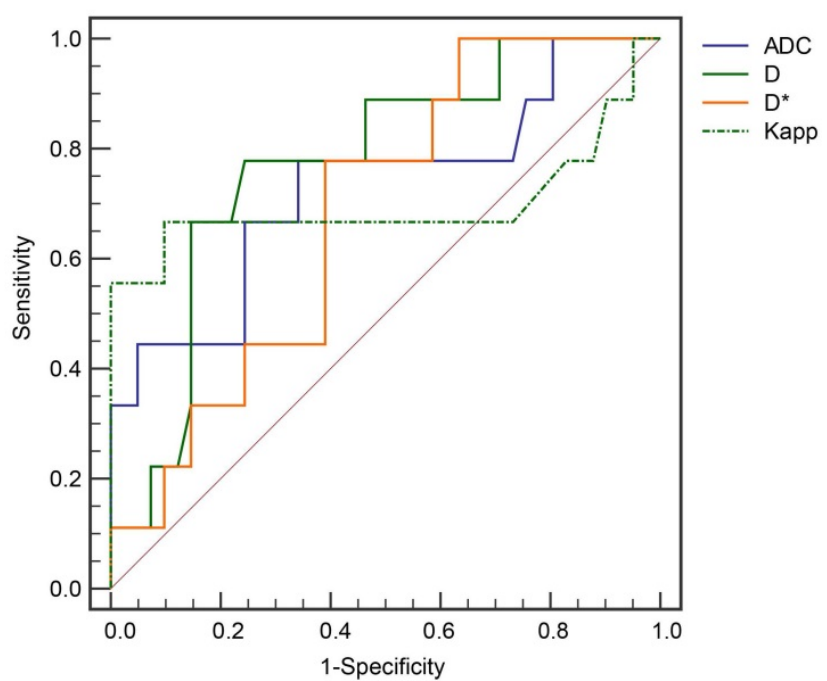

Figure 4. Receiver operating characteristic (ROC) curve analyses. (A) ROC curve analyses show the diagnostic performance of ADC, D, D*, Kapp in distinguishing the high Ki-67 group from the low Ki-67 group. D values produced the highest AUROC of $0.85(95 \% \mathrm{Cl}: 0.73-0.97, P<0.05)$. (B) ROC analysis of ADC, D, Dapp, and Kapp in differentiating SCLC and NSCLC. The $2 \times 2$ contingency analyses, including the cut-off values, sensitivities, specificities, Youden indices, and F1 scores, are listed in Table 3 and Table S4. The raw data are shown in Table S5. ADC: Apparent diffusion coefficient; D: true diffusion coefficient, D* value: perfusion-related pseudodiffusion coefficient; f: perfusion fraction; Kapp: diffusion kurtosis; Dapp: diffusivity; AUROC: Area under ROC curve; Cl: confidence interval; SCLC: small cell lung cancer; NSCLC: non-small cell lung cancer. 
Ki-67 is a common biomarker of tumor cell proliferation and has been shown to be associated with lung cancer prognoses and therapeutic efficacies $[5,31-33]$. In this study, we evaluated the associations between the DWI, IVIM, DKI parameters, and Ki-67 proliferation in tumor tissues of patients with lung cancer. We selected a cut-off of $25 \%$ for Ki-67 expression to differentiate the low and high Ki-67 groups [34]. We found that $\mathrm{D}$ values from IVIM were negatively correlated with Ki-67 expression (Spearman's coefficient $r=-0.76$ ). D values between the high and low Ki-67 expression groups were significantly different. It is likely that the highly proliferative tumors (high Ki-67 expression) had higher inner structural complexities due to increased cellularity, vascular hyperplasia, and necrosis [23].

Although Dapp is corrected for non-Gaussian bias, it showed no significant correlation with Ki-67 expression. This might be due to tumor heterogeneity caused by vascular proliferation and internal necrosis. $D^{*}$ and $f$ are IVIM perfusion-related parameters. No significant correlations were found between the $\mathrm{D}^{*}$ or $\mathrm{f}$ values and Ki-67 expression in lung cancer, consistent with previous bladder cancer and sinonasal tumor studies $[17,18]$. These results indicated that extracellular components, such as average blood velocities, blood microcirculation, and capillary volumes, contributed little to lung tumor aggressiveness. In addition, we found poor $\mathrm{D}^{*}$ and $\mathrm{f}$ value reproducibilities in lung cancer imaging, which might explain their low diagnostic performance in differentiating high and low Ki-67 groups.

$\mathrm{D}$ represents the true molecular diffusion of water without adsorption [14]. In our study, D values performed well to identify lung tumors with high Ki-67 expression. Our results suggest that water molecule diffusion is affected more by tumor proliferation than by the complexity of the tumor microenvironment.

Previous studies [35, 36] have reported that ADC values were significantly different between SCLC and NSCLC. Our study found that ADC, D, and Dapp values of SCLC were dramatically lower than those of NSCLC. The potential histopathologic rationale might be that SCLCs are highly cellular, and the cells have large nuclei with scant cytoplasm; all of these factors could restrict diffusion motion reducing $\mathrm{ADC}, \mathrm{D}$, and Dapp values [37]. As mentioned above, $D^{*}$ and $f$ values were poorly repeatable. $\mathrm{D}^{*}$ values were also related to blood flow velocities. The high variability of $\mathrm{D}^{*}$ values could result from the dramatic differences in tumor vascularities among different tumor types [17]. The $f$ values are affected by the T2 contributions in both the perfusion and pure molecular diffusion compartments. Therefore, measuring IVIM D values could be used as a noninvasive approach to distinguish SCLC and NSCLC.

Our study had several limitations. First, the distribution of lesion types was uneven, and the number of patients with SCLC was relatively small compared with the number of patients with NSCLC. When the dataset was unbalanced, traditional classification metrics, such as AUROCs and sensitivities, might be overfitted. The F1 score is the weighted average of precision and recall; data distribution is considered and particularly suitable for unbalanced datasets [38]. Although the F1 score is acceptable for our analyses, further validations with other independent cohorts are still required. Second, ROIs drawn on the parametric maps might not correspond well with the $\mathrm{Ki}-67$ expression in histologic specimens. MR-guided biopsies could solve this problem. Third, the optimal b-value combination for lung IVIM and DKI analyses was unclear. Adding more $b$ values could improve fitting accuracies, improving IVIM or DKI parameter performance predictions. However, acquisition times would be increased accordingly.

\section{Conclusions}

In summary, $\mathrm{ADC}, \mathrm{D}$, and Kapp values were significantly associated with $\mathrm{Ki}-67$ proliferation statuses in patients with lung cancer. D values obtained from IVIM had the highest diagnostic performance in distinguishing high and low Ki-67 statuses. Moreover, D values to differentiate SCLC and NSCLC performed well. Our study provided a noninvasive approach to predict Ki-67 expression and distinguish different lung cancer types.

\section{Supplementary Material}

Supplementary figures and tables. http://www.jcancer.org/v12p0190s1.pdf

\section{Acknowledgements}

\section{Author contributions}

SL and GL designed and guided the work. YZ, $\mathrm{WH}, \mathrm{XZ}, \mathrm{CL}$, and $\mathrm{CF}$ participated in the work and analyzed the patient data. YZ performed MRI examinations. $Y Z$ and $W H$ were major contributors in writing the manuscript. $X Z, C L$, and $C F$ contributed to Language polishing. $\mathrm{YZ}$ and $\mathrm{WH}$ contributed equally to this article as the co-first authors. All authors read and approved the final manuscript.

\section{Funding}

This study was supported by the General Project from Shanghai Municipal Health Bureau $(\mathrm{CN})$ (201940174). 


\section{Competing Interests}

The authors have declared that no competing interest exists.

\section{References}

1. Torre LA, Bray F, Siegel RL, Ferlay J, Lortet-Tieulent J, Jemal A. Global cancer statistics, 2012. CA Cancer J Clin. 2015; 65: 87-108.

2. Domingues D, Turner A, Silva MD, Marques DS, Mellidez JC, Wannesson L, et al. Immunotherapy and lung cancer: current developments and novel targeted therapies. Immunotherapy. 2014; 6: 1221-35.

3. Molinari C, Clauser P, Girometti R, Linda A, Cimino E, Puglisi F, et al. MR mammography using diffusion-weighted imaging in evaluating breast cancer: a correlation with proliferation index. Radiol Med. 2015; 120: 911-8.

4. Chen WJ, He DS, Tang RX, Ren FH, Chen G. Ki-67 is a valuable prognostic factor in gliomas: evidence from a systematic review and meta-analysis. Asian Pac J Cancer Prev. 2015; 16: 411-20.

5. Wen S, Zhou W, Li CM, Hu J, Hu XM, Chen P, et al. Ki-67 as a prognostic marker in early-stage non-small cell lung cancer in Asian patients: a meta-analysis of published studies involving 32 studies. BMC Cancer. 2015; 15: 520.

6. Wei DM, Chen WJ, Meng RM, Zhao N, Zhang XY, Liao DY, et al. Augmented expression of $\mathrm{Ki}-67$ is correlated with clinicopathological characteristics and prognosis for lung cancer patients: an up-dated systematic review and meta-analysis with 108 studies and 14,732 patients. Respir Res. 2018; 19: 150.

7. Besusparis J, Plancoulaine B, Rasmusson A, Augulis R, Green AR, Ellis IO, et al. Impact of tissue sampling on accuracy of Ki67 immunohistochemistry evaluation in breast cancer. Diagn Pathol. 2016; 11: 82.

8. Juan MW, Yu J, Peng GX, Jun LJ, Feng SP, Fang LP. Correlation between DCE-MRI radiomics features and Ki-67 expression in invasive breast cancer. Oncol Lett. 2018; 16: 5084-90.

9. Matsuda M, Kido T, Tsuda T, Okada K, Shiraishi Y, Suekuni H, et al. Utility of synthetic MRI in predicting the $\mathrm{Ki}-67$ status of oestrogen receptor-positive breast cancer: a feasibility study. Clin Radiol. 2020; 75: 398 e1- e8.

10. Li HM, Liu J, Qiang JW, Gu WY, Zhang GF, Ma FH. Endometrial Stromal Sarcoma of the Uterus: Magnetic Resonance Imaging Findings Including Apparent Diffusion Coefficient Value and Its Correlation With Ki-67 Expression. Int J Gynecol Cancer. 2017; 27: 1877-87.

11. Jiang JX, Zhao JL, Zhang Q, Qing JF, Zhang SQ, Zhang YM, et al. Endometrial carcinoma: diffusion-weighted imaging diagnostic accuracy and correlation with Ki-67 expression. Clin Radiol. 2018; 73: 413.e1-e6.

12. Karaman A, Durur-Subasi I, Alper F, Araz O, Subasi M, Demirci E, et al. Correlation of diffusion MRI with the Ki-67 index in non-small cell lung cancer. Radiol Oncol. 2015; 49: 250-5.

13. Koyama H, Ohno Y, Seki S, Nishio M, Yoshikawa T, Matsumoto S, et al. Value of diffusion-weighted MR imaging using various parameters for assessment and characterization of solitary pulmonary nodules. Eur J Radiol. 2015; 84: 509-15.

14. Le Bihan D, Breton E, Lallemand D, Aubin ML, Vignaud J, Laval-Jeantet M. Separation of diffusion and perfusion in intravoxel incoherent motion MR imaging. Radiology. 1988; 168: 497-505.

15. Kim Y, Ko K, Kim D, Min C, Kim SG, Joo J, et al. Intravoxel incoherent motion diffusion-weighted MR imaging of breast cancer: association with histopathological features and subtypes. Br J Radiol. 2016; 89: 20160140.

16. Lee YJ, Kim SH, Kang BJ, Kang YJ, Yoo H, Yoo J, et al. Intravoxel incoherent motion (IVIM)-derived parameters in diffusion-weighted MRI: Associations with prognostic factors in invasive ductal carcinoma. J Magn Reson Imaging. 2017; 45: 1394-406.

17. Wang $\mathrm{F}, \mathrm{Wu} \mathrm{LM}$, Hua $\mathrm{XL}$, Zhao ZZ, Chen $\mathrm{XX}, \mathrm{Xu}$ JR. Intravoxel incoherent motion diffusion-weighted imaging in assessing bladder cancer invasiveness and cell proliferation. J Magn Reson Imaging. 2018; 47: 1054-60.

18. Xiao Z, Zhong Y, Tang Z, Qiang J, Qian W, Wang R, et al. Standard diffusion-weighted, diffusion kurtosis and intravoxel incoherent motion MR imaging of sinonasal malignancies: correlations with Ki-67 proliferation status. Eur Radiol. 2018; 28: 2923-33.

19. Rosenkrantz AB, Padhani AR, Chenevert TL, Koh DM, De Keyzer F, Taouli B, et al. Body diffusion kurtosis imaging: Basic principles, applications, and considerations for clinical practice. J Magn Reson Imaging. 2015; 42: 1190-202.

20. Jensen JH, Helpern JA, Ramani A, Lu H, Kaczynski K. Diffusional kurtosis imaging: the quantification of non-gaussian water diffusion by means of magnetic resonance imaging. Magn Reson Med. 2005; 53: 1432-40.

21. Jiang R, Jiang J, Zhao L, Zhang J, Zhang S, Yao Y, et al. Diffusion kurtosis imaging can efficiently assess the glioma grade and cellular proliferation. Oncotarget. 2015; 6: 42380-93.

22. Shi RY, Yao QY, Zhou QY, Lu Q, Suo ST, Chen J, et al. Preliminary study of diffusion kurtosis imaging in thyroid nodules and its histopathologic correlation. Eur Radiol. 2017; 27: 4710-20.

23. Sun $\mathrm{K}$, Chen $\mathrm{X}$, Chai W, Fei X, Fu C, Yan X, et al. Breast Cancer: Diffusion Kurtosis MR Imaging-Diagnostic Accuracy and Correlation with Clinical-Pathologic Factors. Radiology. 2015; 277: 46-55.
24. Nguyen XC, Lee WW, Chung JH, Park SY, Sung SW, Kim YK, et al. FDG uptake, glucose transporter type 1, and Ki-67 expressions in non-small-cell lung cancer: correlations and prognostic values. Eur J Radiol. 2007; 62: 214-9.

25. Kaira K, Oriuchi N, Imai H, Shimizu K, Yanagitani N, Sunaga N, et al. Prognostic significance of L-type amino acid transporter 1 expression in resectable stage I-III nonsmall cell lung cancer. Br J Cancer. 2008; 98: 742-8.

26. Maddau C, Confortini M, Bisanzi S, Janni A, Montinaro F, Paci E et al. Prognostic significance of p53 and Ki-67 antigen expression in surgically treated non-small cell lung cancer: immunocytochemical detection with imprint cytology. Am J Clin Pathol. 2006; 125: 425-31.

27. Yu L, Li K, Li X, Guan C, Sun T, Zhang X. Metabolomic profiling of dried blood spots reveals gender-specific discriminant models for the diagnosis of small cell lung cancer. Aging (Albany NY). 2020; 12: 978-95.

28. Han I, Xia Y, Lin L, Zhang Z, Tian H, Li K. Next-generation Metabolomics in the Development of New Antidepressants: Using Albiflorin as an Example. Curr Pharm Des. 2018; 24: 2530-40.

29. Li K, Naviaux JC, Monk JM, Wang L, Naviaux RK. Improved Dried Blood Spot-Based Metabolomics: A Targeted, Broad-Spectrum, Single-Injection Method. Metabolites. 2020; 10.

30. E L, Lu L, Li L, Yang H, Schwartz LH, Zhao B. Radiomics for Classifying Histological Subtypes of Lung Cancer Based on Multiphasic Contrast-Enhanced Computed Tomography. J Comput Assist Tomogr. 2019; 43: 300-6.

31. Ishibashi N, Maebayashi T, Aizawa T, Sakaguchi M, Nishimaki H, Masuda S. Correlation between the $\mathrm{Ki}-67$ proliferation index and response to radiation therapy in small cell lung cancer. Radiat Oncol. 2017; 12: 16.

32. Tabata K, Tanaka T, Hayashi T, Hori T, Nunomura S, Yonezawa S, et al. Ki-67 is a strong prognostic marker of non-small cell lung cancer when tissue heterogeneity is considered. BMC Clin Pathol. 2014; 14: 23.

33. Warth A, Cortis J, Soltermann A, Meister M, Budczies J, Stenzinger A, et al. Tumour cell proliferation (Ki-67) in non-small cell lung cancer: a critical reappraisal of its prognostic role. Br J Cancer. 2014; 111: 1222-9.

34. Tong G, Zhang G, Liu J, Zheng Z, Chen Y, Niu P, et al. Cutoff of $25 \%$ for Ki67 expression is a good classification tool for prognosis in colorectal cancer in the AJCC8 stratification. Oncol Rep. 2020; 43: 1187-98.

35. Koyama H, Ohno Y, Nishio M, Takenaka D, Yoshikawa T, Matsumoto S, et al. Diffusion-weighted imaging vs STIR turbo SE imaging: capability for quantitative differentiation of small-cell lung cancer from non-small-cell lung cancer. Br J Radiol. 2014; 87: 20130307.

36. Liu H, Liu Y, Yu T, Ye N. Usefulness of diffusion-weighted MR imaging in the evaluation of pulmonary lesions. Eur Radiol. 2010; 20: 807-15.

37. Razek AA, Fathy A, Gawad TA. Correlation of apparent diffusion coefficient value with prognostic parameters of lung cancer. J Comput Assist Tomogr. 2011; 35: 248-52.

38. Chicco D, Jurman G. The advantages of the Matthews correlation coefficient (MCC) over F1 score and accuracy in binary classification evaluation. BMC Genomics. 2020; 21: 6 . 